\title{
Toxicidad de piel
}

\section{Toxicidad de piel. Prevencion y tratamiento}

R. Esco, J. Valencia, S. Polo, M. López Mata

\section{Introducción}

Actualmente la toxicidad cutánea por irradiación está disminuyendo por los avances técnicos que se han dado en el campo de la radioterapia, tales como energías altas cuya zona de equilibrio electrónico se encuentra en muchos casos, no sólo a milímetros, sino a centímetros de profundidad, así como por la utilización de modernas técnicas de planificación, que permiten utilizar múltiples puertas de entrada y de esta manera repartir la dosis que pueda recibir la piel. No obstante, la piel sigue siendo la puerta de entrada de toda radiación y por tanto el primer lugar de agresión.

Por otro lado, los oncólogos radioterápicos siempre hemos intentado cuidar la piel al máximo, pues si bien, en general, la toxicidad cutánea no es especialmente grave, es molesta para el paciente.

\section{Prevención o tratamiento}

A la hora de plantear el cuidado de la piel, la primera pregunta que debemos hacernos es si existe prevención efectiva o si debemos esperar a que aparezcan los efectos secundarios y entonces tratarlos.

Si revisamos la bibliografía referente a toxicidad cutánea y su manejo, vemos que, en general, durante los años 50-70 los artículos publicados eran de tratamientos de la toxicidad ya establecida. Igualmente estos primeros trabajos usaban corticoides y eran observacionales. Los pocos comparativos comparaban eficacia de 2 corticoides, pues se daba por hecho que el tratamiento de la lesión cutánea se realizaba mediante el empleo de corticoterapia tópica.

En las dos últimas décadas prácticamente todos los artículos publicados se refieren al empleo de diversos agentes para prevenir la toxicidad cutánea y al ser trabajos de prevención todos deben ser randomizados, en los que se compara

Servicio de Oncología Radioterápica

Hospital Clínico Lozano Blesa y Quirón La Floresta Zaragoza la efectividad de diversos productos, fundamentalmente sustancias emolientes, (son sustancias blandas grasas u oleosas que hidratan y suavizan la piel, son oclusivas y suelen contener parafina), en general frente a placebo, para verificar la capacidad de ellos para evitar, retrasar o disminuir el grado de lesión cutánea radioinducida.

Por ello, parece claro que la tendencia actual es a prevenir la toxicidad cutánea.

\section{Tratamiento de la dermitis por irradiación Corticoides}

En el tratamiento de la dermitis por irradiación se han usado fundamentalmente los corticoides tópicos. Su mecanismo de acción es a nivel de la reacción inflamatoria que en la dermis se produce como consecuencia del depósito de energía que la radiación produce. Esta energía induce cambios consistentes en vasodilatación, con aparición de enrojecimiento, aumento de la permeabilidad vascular, proliferación de leucocitos y facilitación de su migración. Los corticoides actúan a estos cuatro niveles con reducción de la vasodilatación, lo que condiciona una reducción de la permeabilidad capilar, inhibición de la proliferación leucocitaria y de su migración.

Los primeros trabajos datan de 1954 y son de Houghton' ${ }^{1} y$ posteriormente en 1965 de Biörnberg². En 1979, Gless ${ }^{3}$ presenta un ensayo en el que randomiza a 54 pacientes, la mitad se tratan con hidrocortisona y la otra mitad con clobetasona, la administración se comienza cuando los pacientes alcanzan la dosis de $20 \mathrm{~Gy}$, independientemente del grado de dermatitis. La conclusión es que no se observa reducción de la reacción cutánea esperada y que los pacientes con clobetasona tienen mayor grado de dermatitis que los tratados con hidrocortisona. Por otro lado los tratamientos con corticoides tópicos inducen la aparición de atrofias cutáneas tardías, por lo que los autores concluyen que no deben ser usados.

Schmuth ${ }^{4}$ en un estudio randomizado comparando la eficacia como preventivo de metilprednisolona tópica frente a dexpantenol (hidratante), concluye que no se observa prevención con ninguno de los 2 productos, si bien el grado de afectación cutánea es menor en el grupo de corticoides. Böstrom ${ }^{5}$ en un estudio randomizado en el que compara mometasona frente a hidratante en la prevención de la dermatitis por irradiación, llega a la conclusión de que aparece una 
TABLA I

Emolientes e higiene en la prevención de toxicidad aguda cutánea

\begin{tabular}{llll}
\hline Autor & Agente & Pac. & Resultados \\
\hline Olsen (11) & Jabon vs Jabón + aloe & 50 & $\begin{array}{l}\text { 27 Gy: SIN DIF } \\
\text { 27 Gy: 3 vs 5 sem. } \\
\text { Roy (12) }\end{array}$ \\
Fisher (13) & Lavarse vs No & & SIN DIFERENCIAS \\
Cerezo (14) & Biafine vs nada & SIN DIFERENCIAS. \\
& Sativa vs nada & 172 & Eritema : 72 vs 100 \% \\
Heggie (15) & Hidratante vs aloe & 50 & Reduce: grado y tiempo cicatrización. \\
Martin (16) & Aloe vs nada & & MEJOR ALOE \\
Momm (17) & Hidrat vs hid+urea & 225 & MEJOR HID+UREA \\
Graham (18) & Hidratante vs gel (3M Cavilon) & 30 & MEJOR GEL: Descamación: 33 vs 46 \\
& & 88 & Reduce prurito y durac. \\
\hline
\end{tabular}

reducción en el grado de eritema en el grupo tratado con mometasona, aunque en la valoración de la pigmentación postirradiación no encuentra diferencias estadísticamente significativas.

En resumen podría concluirse que los corticoides son activos en el tratamiento de la dermatitis por irradiación y que reducen la intensidad del eritema si se emplean como preventivos. No obstante tienen efectos secundarios que puede limitar su uso.

\section{Prevención de la toxicidad cutánea}

Además de los estudios mencionados en los que se emplea corticoides tópicos, se han usado otras sustancias como preventivas de la toxicidad, entre ellas citaremos:

\section{Sucralfato}

Sucralfato tiene capacidad antiulcerosa, activa la proliferación celular y tiene una cierta actividad antiinflamatorio, pensándose que es a estos tres niveles como puede actuar a nivel de la dermitis radioinducida.

Los resultados con sucralfato son contradictorios. Delaney ${ }^{6}$ en un estudio randomizado con 39 pacientes en el que compara un emoliente (sorbolene) versus el mismo emoliente con $10 \%$ de sucralfato, comenzándolo a emplear cuando se presenta la reacción cutánea. Los autores no encuentran diferencias entre ambos grupos.

Maiche $^{7}$ en un estudio randomizado doble ciego, en el que compara una crema base versus la misma con sucralfato en 50 pacientes tratadas con electrones en pared costal, concluye que las pacientes tratadas con sucralfato tienen una reducción del grado de reacción cutánea y la recuperación es mas rápida en el grupo de sucralfato.

Por el contrario, Wells ${ }^{8}$ no encuentra diferencias estadísticamente significativas entre sucralfato, empleo de hidratantes cutáneos o nada como preventivo de toxicidad, en un estudio entre 375 pacientes.

\section{Higiene y emolientes}

El empleo de sustancias hidratantes o medidas tendentes a mejorar la hidratación de la piel han comenzado a ver la luz de las publicaciones a partir del año 2000. Estas sustancias se están empleando con carácter profiláctico, por lo que necesariamente las publicaciones deben ser estudios comparativos entre la sustancia a investigar, la observación u otra. Ocasionalmente se ha publicado algún artículo observacional en el que, sin ser el objetivo principal del trabajo, se utiliza algún producto hidratante como coadyuvante al tratamiento principal y se muestran sus resultados enfrentándolos a los resultados ya publicados y conocidos

En esta línea, Porok ${ }^{9}$ y Szumacher ${ }^{10}$ han publicado 2 estudios observacionales concluyendo que no parece mejor la aparición de toxicidad cutánea, aunque mejora el confort de los pacientes. No obstante, es difícil obtener conclusiones de estudios observacionales en los que se pretende demostrar la efectividad de un tratamiento preventivo, pues la aparición y grado de dermatitis está sometida a muchas variables radioterápicas que hacen difícil la valoración.

Respecto a estudios randomizados, en la Tabla I se muestra un resumen de lo publicado en la literatura. Como puede apreciarse los resultados son bastante contradictorios nuevamente. Llama la atención la discrepancia que puede existir respecto a una buena higiene local, así Roy ${ }^{12}$ no encuentra diferencias en una serie de 99 pacientes en los que compara la dermatitis respecto a la única variable de lavarse cuidadosamente la piel o no. La toxicidad grado 0 es del $2 \%$ en el grupo que no se lava, frente al 0\%. Para grado I es de 41 verus $64 \%$ (Control versus lavado) y para grado II es del 57 versus $34 \%$, pero estas diferencias no son estadísticamente significativas. Respecto a la utilización de jabón versus la misma higiene y aplicación de un gel de Aloe Vera, Olsen ${ }^{11}$, tampoco encuentra diferencias, mientras que entre nosotros, tanto Cerezo ${ }^{14}$ como Martin ${ }^{16}$, si que encuentran mejores resultados, Cerezo con la utilización de lavado y crema hidratante y Martin con el empleo de Aloe Vera. Para confundir más los resultados Heggie ${ }^{15}$ publica peores resultados al comparar el empleo de un producto hidratante frente a gel de Aloe Vera con el empleo de Aloe Vera.

\section{Ácido hialurónico}

Se han empleado otros productos en la prevención de la toxicidad cutánea, entre ellos el ácido hialurónico. Se espe- 


\section{R. Escó y cols.}

cula que su mecanismo de acción sea fundamentalmente antiinflamatorio, además de favorecer la formación tejido de granulación y la remodelación de la matriz extracelular.

Ligouri $^{19}$ en un ensayo randomizado doble ciego en el que incluye 152 pacientes comparando crema de ácido hialurónico con placebo, encuentra que el grupo de pacientes que reciben la crema con ácido hialurónico presentan una reducción de la intensidad de la dermitis y un retardo en el tiempo de aparición de esta, de manera estadísticamente significativa, así como una valoración subjetiva de mayor confort en el grupo de ácido hialurónico.

\section{Antiestrés oxidativo}

La irradiación libera radicales oxígeno que son responsables en parte de los fenómenos inflamatorios agudos producidos por la irradiación. La inhibición de estos radicales mediante dismutadores de ellos podría beneficiar a estos pacientes como se han demostrado en otras localizaciones ${ }^{20,21}$.

En piel se han publicado resultados con Theta $\mathrm{crem}^{22}$, que contiene como antioxidante C M Glucan, sin que en la serie de 20 pacientes se hallan encontrado diferencias estadísticamente significativas.

Entre nosotros, el grupo del Hospital General de la Defensa está trabajando con superóxido dismutasa en crema para dermatitis con irradiación, con resultados pendientes de publicar de manera definitiva.

\section{Otros}

Se han empleado multitud de productos en la prevención de toxicidad cutánea, algunos sin más interés que la curiosidad, entre ellos un estudio de Lokkevik ${ }^{23}$ en el que emplea una crema con vitamina $B$, sin encontrar diferencias.

A nivel experimental ${ }^{24}$, se han empleado dietas ricas en ácido linoléico y eicosapentaenóico, con buenos resultados, en cerdos.

\section{Resumen}

A modo de resumen Wickline (25) revisa 18 artículos que tratan sobre el tema, publicados entre 1967 y 2001. Las conclusiones a las que llega son: que diversos productos como la crema de camomila, la vitamina C tópica, el aceite de almendras y el violeta de genciana que se empleó en los años 50 y 60, no han mostrado ninguna utilidad.

Los hidrocoloides, sucralfato y corticoides son beneficiosos.

Los geles con Aloe Vera pueden ser beneficiosos.

Pero como conclusión afirma que actualmente no existe evidencia científica que permita dar una guía de uso clínico para prevención y tratamiento de la dermatitis por irradiación.

Tal vez una revisión sistemática de la literatura y la realización de un metaanálisis de los resultados, algunos tan dispares existentes, permita obtener conclusiones con nivel de evidencia mayor.

\section{Bibliografía}

1. Houghton $L E$, Walter JB, Jones DEA. The inhibition of acute $X$ ray damage by cortisone. $\mathrm{Br}$ Med J 1954; 2:1313-1315.

2. Björnberg A Hellgren L, Olsson S. Treatment of radiation dermirtis with fluocinolone acetonide. Acta Radiother Phys Biol $1965 ; 3: 129-133$.

3. Gless JP, Mameghan-Zadeh H, Sparkes CG. Effectiveness of topical steroids in the control of radiation dermatitis: a randomised trial using $1 \%$ hydrocortisone cream and $0.05 \%$ clobetasone butyrate (Emovate). Clin Radiol. 1979; 30(4): 397-403.

4. Schmuth $M$, Wimmer MA, Hofer $S$,el al. Topical corticosteroid therapy for acute radiation dermatitis: a prospective, randomized, double-blind study. Br J Dermatol 2002 Jun; 146(6): 983-991.

5. Böstrom $A$, Lindman $H$, Swartling $C$ et al. Potent corticosteroid cream (mometasonenfuroate) significantly reduces acute radiation ermatitis: results from double-blind, randomized study. Radiother Oncol 2001 Jun; 59(3): 257-265.

6. Delaney G, Fisher R Hook C, Barton M. Sucralfate cream in the management of moist desquamation during radiotherapy. Australas Radiol. 1997 Aug; 41 (3): 270-275.

7. Maiche A, Isokangas OP, Grohn P. Skin protection by sucralfate cream during electron beam therapy. Acta Oncol 1994; 33(2): 201-203.

8. Wells $M$, Macmillan $M$, Raab $G$, et al. Does aqueous or sucralfate cream effect the severity of erythematous radiation skin reactions? A randomised controlled trial. Radiother Oncol. 2004 Nov; 73: 153-162.

9. Porok D, Kristjanson L. Skin reactions during radiotherapy for breast cancer: the use and impacto $f$ topical agents and dressings Eur J Cancer Care (Eng) 1999 Sep; 8(3): 143-153.

10. Szumacher E, Wighton A, Franssen $E$, et al. Phase II study assessing the effectiveness of Biafine cream as a prophylactic agent for radiation-induced acute skin toxicity to the breast in women undergoing radiotherapy with concomitant CMF chemotherapy Int J Radiat Oncol Biol Phys 2001; 51 (1): 81-86.

11. Olsen DI, Raub W, Bradley et al. The effect of aloe vera $\mathrm{gel} /$ mild soap versus mild soap alone in preventing skin reactions in patients undergoing radiation therapy. Oncol Nurs Forum. 2001 Apr;28 (3): 543-547.

12. Roy I, Fortin A, Larochelle M. The impact of the skin washing with water and soap during breast irradiation: a randomized study. Radiother Oncol. 2001 Mar; 58(3): 333-339.

13. Fisher J, Scott $C$, Stevens $R$, et al. Randomized phase II study Best Supportive Care to Biafine as a prophylactic agent for radiation-induced skin toxicity for women undergoing breast irradiation: Radiation Therapy Oncolgy Group (RTOG) 97-13. Int J Radiat Oncol Biol Phys. 2000 Dec 1; 48(5): 1307-1310.

14. Cerezo L, Iglesias A, Chinchetru $C$, et al. Efectos del lavado e hidratación de la piel durante el tratamiento radioterápico: Resultados de un estudio randomizado. Rev Oncología 2001; 3(supl 1):203.

15. Heggie S, Bryant GP, Tripcony L, et al. A phase III study on the efficacy of topical aloe vera gel on irradiated breast tissue. Cancer Nurs 2002 Dec; 25(6): 442-451.

16. Martin A, Iglesias A, Chinchetru $C$, et al. Estudio de la respuesta dérmica con Aloe Vera en el tratamiento radioterápico del cancer de mama. Rev Oncología 2003; 5(supl 4):26.

17. Momm F, Weissenberger C, Bartelt S, Henke M. Moist skin care can diminish acute radiation-induced skin toxicity Strahlenther Onkol 2003 Oct; 179(10): 708-712. 
18. Graham P, Browne L, Capp A, et al. Randomized, paired comparison of No-Sting Barrier Film versus sorbolene cream (10\% glyceine) skin care during postmastectomy irradiation. Int J Radiat Oncol Biol Phys. 2004 Jan 1; 58(1): 241-246.

19. Ligouri V, Guillemin C, Pesce GF, et al. Double-blind, randomized clinical study comparing hyaluronic acid cream to placebo in patients treated with radiotherapy. Radiother Oncol. 1997 Feb; 42(2): 155-161.

20. Esco R, Valencia J, Coronel $P$, et al. Efficacy of orgotein in prevention of late side effects of pelvic irradiation: a randomized study. Int J Radiat Oncol Biol Phys. 2004 Nov 15; 60(4): 1211-1219.

21. Valencia J, Velilla J, Urpegui J, et al. The efficacy of orgotein in the treatment of acute toxicity due to radiotherapy on head and neck fumors. Tumori 2002; 88:385-389.
22. Roper B, Kaisig D, Auer F, et al. Theta cream versus Bepanthol lotion in breast cancer patients under radiotherapy: A new prophylactic agent in skin care? Strahlenther Onkol. 2004 May; 180(5): 315-322

23. Lokkevik E, Skovlund E, Reitan JB, et al. Skin treatment with bepanthen cream versus no cream during radiotherapy. A randomized controlled trial. Acta Oncol. 1996; 35(8):1021-1026.

24. Hopewell JW, van der Aardweg GJ, Morris GM, et al. Amelioration of both early and late radiation-induced damage to pig skin by essential fatty acids. Int J Radiat Oncol Biol Phys. 1994 Dec 1; 30(5): $1119-1125$.

25. Wickline MM. Prevention and treatment of acute radiation dermatitis: a literatura review. Oncol Nurs Forum 2004 Mar-Apr; 31(2): 237-247. 